\title{
Production of lentiviral vectors with enhanced efficiency to target dendritic cells by attenuating mannosidase activity of mammalian cells
}

April Tai, Steven Froelich, Kye-II Joo, Pin Wang*

\begin{abstract}
Background: Dendritic cells (DCs) are antigen-presenting immune cells that interact with $T$ cells and have been widely studied for vaccine applications. To achieve this, DCs can be manipulated by lentiviral vectors (LVs) to express antigens to stimulate the desired antigen-specific T cell response, which gives this approach great potential to fight diseases such as cancers, HIV, and autoimmune diseases. Previously we showed that LVs enveloped with an engineered Sindbis virus glycoprotein (SVGmu) could target DCs through a specific interaction with DC-SIGN, a surface molecule predominantly expressed by DCs. We hypothesized that SVGmu interacts with DC-SIGN in a mannose-dependent manner, and that an increase in high-mannose structures on the glycoprotein surface could result in higher targeting efficiencies of LVs towards DCs. It is known that 1-deoxymannojirimycin (DMJ) can inhibit mannosidase, which is an enzyme that removes high-mannose structures during the glycosylation process. Thus, we investigated the possibility of generating LVs with enhanced capability to modify DCs by supplying DMJ during vector production.

Results: Through western blot analysis and binding tests, we were able to infer that binding of SVGmu to DC-SIGN is directly related to amount of high-mannose structures on SVGmu. We also found that the titer for the LV (FUGW/SVGmu) produced with DMJ against 293T.DCSIGN, a human cell line expressing the human DC-SIGN atnibody, was over four times higher than that of vector produced without DMJ. In addition, transduction of a human DC cell line, MUTZ-3, yielded a higher transduction efficiency for the LV produced with DMJ.

Conclusion: We conclude that LVs produced under conditions with inhibited mannosidase activity can effectively modify cells displaying the DC-specific marker DC-SIGN. This study offers evidence to support the utilization of DMJ in producing LVs that are enhanced carriers for the development of DC-directed vaccines.
\end{abstract}

\section{Background}

Dendritic cells (DCs) are immune cells that are able to present antigens to $\mathrm{T}$ cells in a major histocompatibility complex (MHC)-restricted manner. These antigens are usually obtained by phagocytosis of pathogens encountered by the DCs [1]. The naive T cells are activated by the interaction with the antigen-presenting DCs and are then able to recognize the corresponding pathogens. To utilize this mechanism for therapeutic applications such as immunizations and vaccinations, DCs can be loaded with antigens to stimulate antigen-specific CD8+ and CD4+ T cell responses [1-4]. Another method of

\footnotetext{
* Correspondence: pinwang@usc.edu

Mork Family Department of Chemical Engineering and Materials Science, University of Southern California, Los Angeles, CA 90089, USA
}

modifying DCs to present desired antigens is to genetically alter the cells by using liposomes or gene-gun, or by viral transduction with replication-incompetent viral vectors $[5,6]$. The benefits of these strategies are the increased time of antigen presentation, the ability to present both MHC I and II epitopes, and the ability to include genes for immomodulatory molecules that may enhance DC function [7]. Currently, adenoviral, gammaretrovial, and lentiviral vectors (LVs) are studied for the viral vector delivery strategy [8-11]. LVs pose an advantage in their ability to transduce non-dividing cells, which is beneficial for in vivo immunization [12-16]. However, these recombinant viral vectors are known to have broad specificity and are able to transduce multiple cell types,

\section{() Biomed Central}


which can inevitably result in genetic modification of undesired cells and reduce vaccine efficacy $[17,18]$.

A surface molecule present on immature DCs, Dendritic Cell-specific ICAM3-grabbing Nonintegrin (DC-SIGN), is well-displayed and a suitable target for DC-specific transduction [18,19]. DC-SIGN is a C-type $\left(\mathrm{Ca}^{2+}\right.$-dependent) lectin that is able to rapidly bind to and endocytose antigenic materials [20]. It is a type II transmembrane protein that is displayed as a tetramer, and consists of a short, $\mathrm{N}$-terminal cytoplasmic tail that contains intracellular sorting motifs, a transmembrane region, an extracellular stalk, and a C-terminal carbohydrate-recognition domain (CRD) [21-23]. It was reported that the Sindbis virus (SV), a member of the alphavirus genus and the Togaviridae family, is able to recognize and bind to DCs through DC-SIGN [24]. However, the SV glycoprotein (SVG) also has the ability to bind to cellsurface heparin sulfate (HS), which is expressed by many cell types, and therefore LVs pseudotyped with SVG have a broad tropism $[25,26]$. Further studies showed that the HS binding site of SVG can be mutated [27] so that the resulting SVGmu glycoprotein can selectively recognize and bind to DC-SIGN [28]. Thus, SVGmu-pseudotyped LVs can specifically target and recognize DCs, delivering antigens that enable $\mathrm{T}$ cell activation for immunization and vaccine purposes [28-30].

The study of DC-SIGN binding to other proteins has shown that binding occurs in a carbohydrate-dependent manner [20,31]; in fact, Sindbis viruses produced in mosquito cells, which limit glycoprotein processing and carbohydrate trimming, yielded higher transduction efficiencies for DC-SIGN-bearing cells compared to viruses produced in mammalian cells [24]. The high-mannose structures on gp120 have also been studied and have been determined to be critical for recognition to DCSIGN [32,33]. Mannosidase is a calcium-dependent enzyme that removes mannose from N-linked glycoproteins in the ER and Golgi [34]. 1-deoxymannojirimycin (DMJ, Figure 1A) is a chemical that can inihibit $\alpha$-mannosidase I in the Golgi by binding to the top of its $\mathrm{C}$-terminal $\alpha$-hairpin, which is located at the bottom of the active site cavity [34-36]. This effectively halts the processing of the oligosaccharide at $\mathrm{Man}_{9} \mathrm{GlcNAc}_{2}$ (Figure 1B) [37]. It has been reported that DC-SIGN

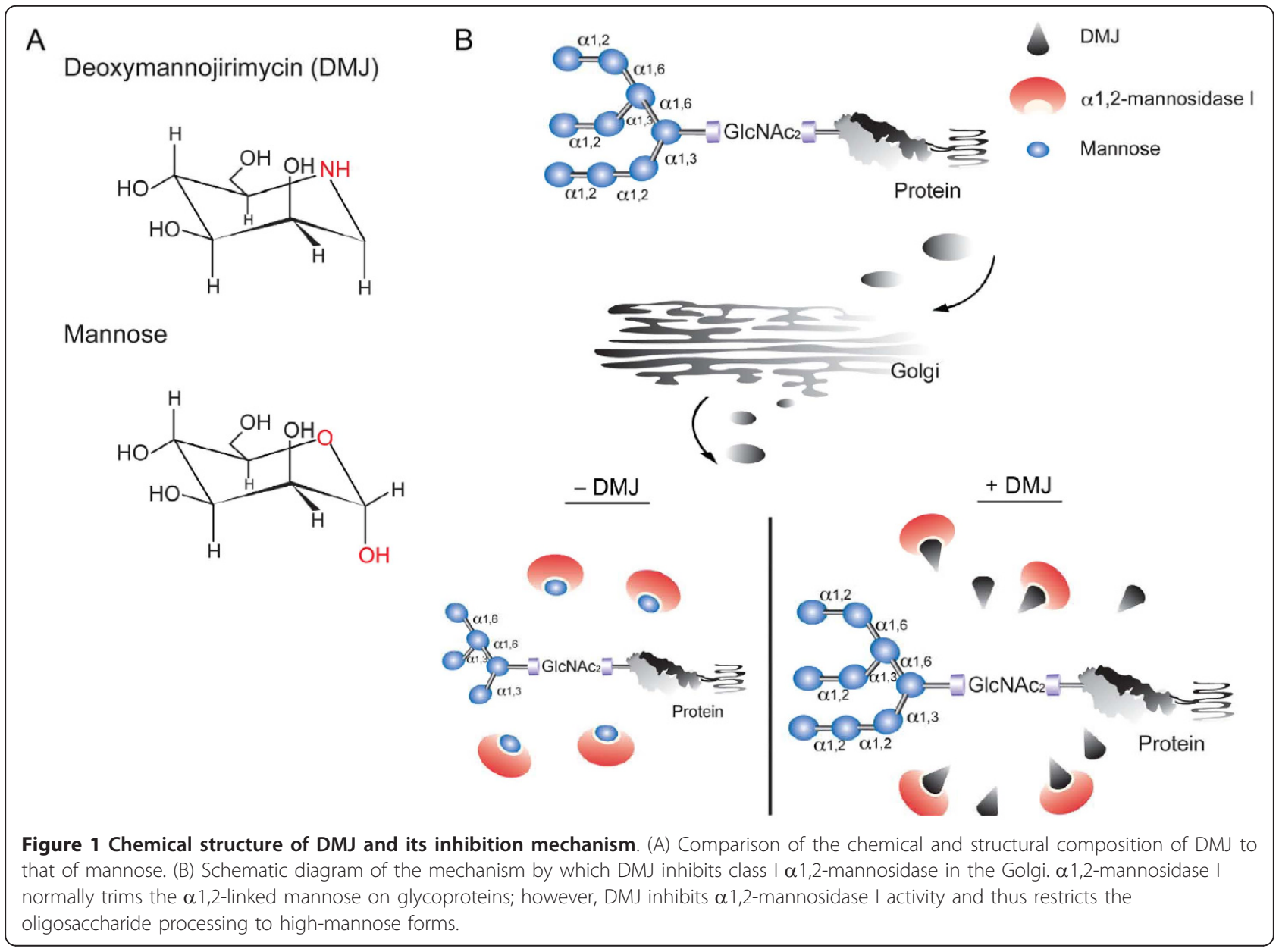


binds to $\mathrm{Man}_{9} \mathrm{GlcNAc}_{2}$ 130-fold more tightly than it does to mannose [31]. Thus, we hypothesized that SVGmu also binds to DC-SIGN through high-mannose structures, and that the addition of DMJ inhibits the activities of $\alpha 1,2$-mannosidase I, which would allow for a greater amount of high-mannose structures on the surface of SVGmu-pseudotyped LVs. In this study, we test this hypothesis and show that SVGmu-bearing LVs produced under DMJ treatment can modify cells expressing the DC-specific marker DC-SIGN more efficiently.

\section{Results and Discussion}

Transient transfection of 293T cells to produce SVGmu-pseudotyped LVs

To assess the viability of producing LVs in media containing DMJ, 293T cells were transiently transfected with a lentiviral backbone plasmid (FUGW) encoding a green fluorescent protein (GFP) reporter gene driven by the human ubiqutin-C promoter [38], packaging plasmids, and a plasmid encoding either SVGmu or vesicular stomatitis virus glycoprotein (VSVG). VSVG has widely been used to pseudotype LVs and the resulting vectors have a very broad tropism [26]. Thus, we included VSVG in our transfection to produce a control vector that is not DC-SIGN-targeting. Analysis of the transfected cells two days later by flow cytometry showed comparable results between the samples with and without DMJ added, with slightly higher values for SVGmu-staining in the cells transfected with SVGmu and cultured with DMJ (Figure 2). Cells transfected with SVGmu stained positively for SVGmu and expressed GFP, while the control cells transfected with VSVG

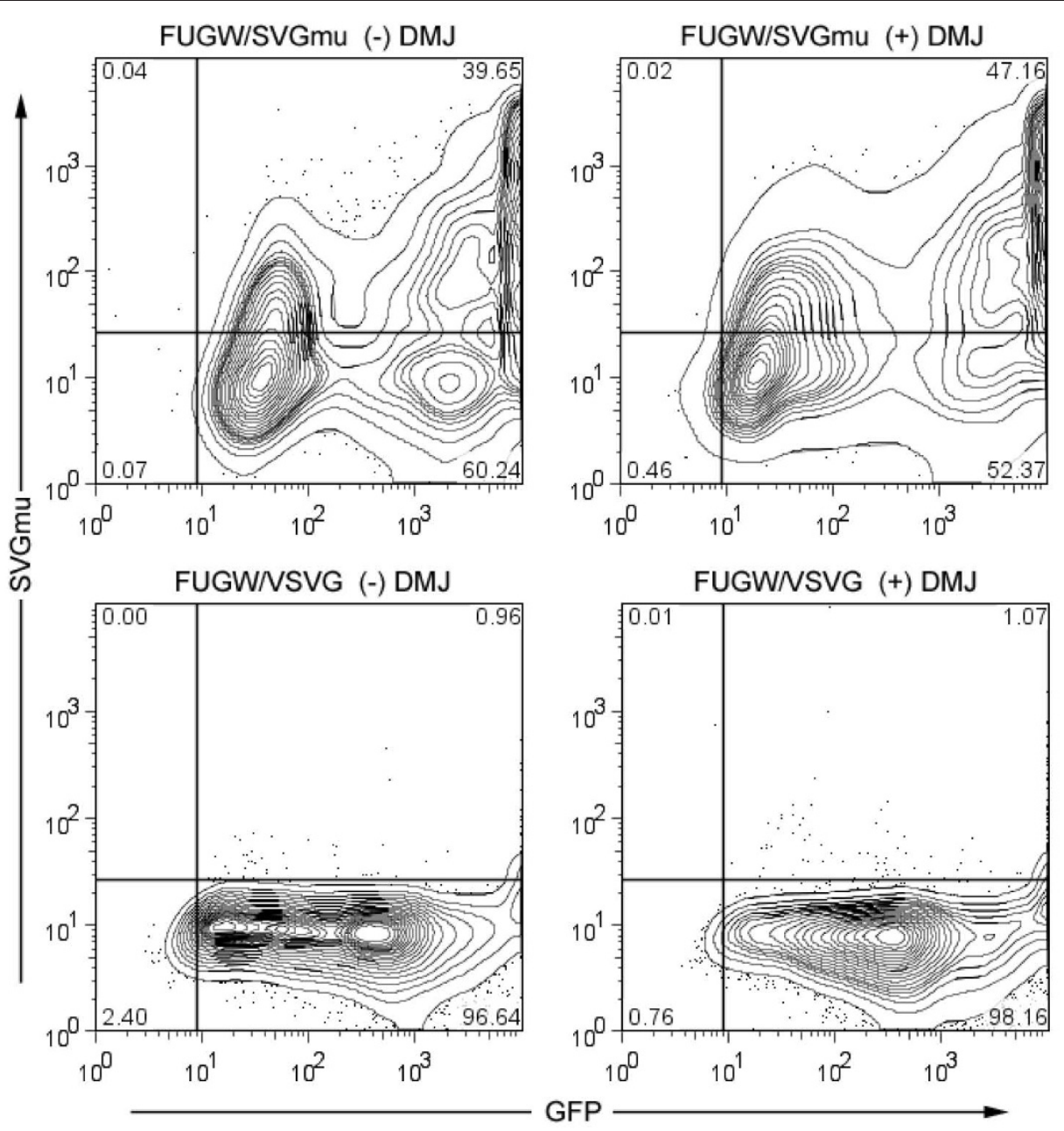

Figure 2 DMJ does not reduce SVGmu production or display on vector-producing cells. 293T cells were transiently transfected by the lentiviral backbone vector encoding the GFP gene (FUGW), packaging constructs (REV and RRE), and a plasmid encoding either SVGmu or VSVG. SVGmu-staining and flow cytometry analysis of the cells two days post-transfection revealed that cells cultured with or without DMJ exhibited similar levels of SVGmu and GFP, indicating that the presence of DMJ did not restrict either glycoprotein or LV production. Control cells transfected by VSVG were similarly unaffected by DMJ and SVGmu-negative, as expected. 
were only GFP-positive. These results indicate that the addition of DMJ to the cell culture media does not adversely affect the transfection of 293T cells.

\section{Verification of SVGmu and high-mannose oligosaccharides on the vector surface}

To verify the presence of SVGmu on the vector particle surface produced in media containing DMJ, we employed a labeling scheme to generate vectors that encapsulated GFP-Vpr (GFP fused with the HIV accessory protein Vpr [39]). A transient co-transfection protocol similar to what was described above, was utilized to generate the GFP-labeled particles, except that the lentiviral backbone plasmid FUW, which lacks the GFP reporter gene, replaced FUGW, and an additional plasmid encoding GFP-Vpr was included in the co-transfection procedure
[39]. The resulting vectors (FUW-GFP-Vpr/SVGmu +/DMJ or FUW-GFP-Vpr/VSVG) were loaded onto cover slips, stained for SVGmu, and analyzed by confocal imaging. The SVGmu envelope glycoprotein was confirmed to be displayed on the FUW-GFPVpr/SVGmu vectors produced both with and without DMJ by the colocalization of the GFP and SVGmu signals (Figure 3). As a control, FUW-GFPVpr/VSVG vector particles were also analyzed. As expected, while these particles were GFPpositive, they did not stain for SVGmu.

Next, the SVGmu-enveloped vector (FUGW/SVGmu) produced either with or without DMJ was concentrated by ultracentrifugation and then digested by EndoH, an enzyme that selectively breaks apart high-mannose structures by cleaving the chitobiose core from N-linked glycoproteins [20]. A western blot analysis of the EndoH-

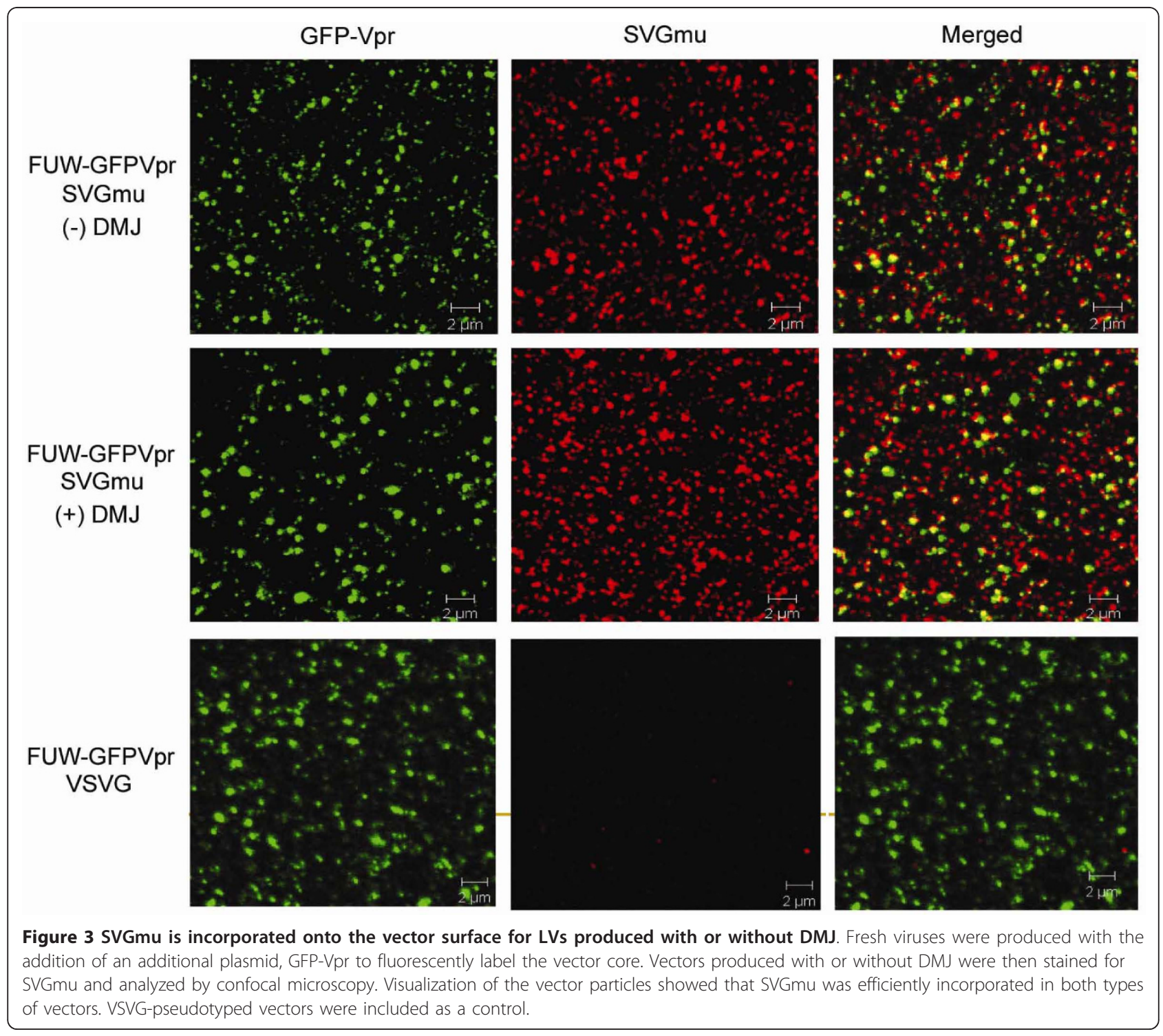


treated vector particles confirmed the presence of highmannose structures for the LVs produced with DMJ by yielding a lower molecular weight species (Figure 4). In contrast, the vector produced without DMJ did not give as low of a molecular weight species when digested by EndoH, which is evidence of less high-mannose structures present on the vector surface. We believe that this supports our hypothesis that the untreated vector contains mostly complex sugars on the envelope glycoprotein, along with some hybrid sugars (both mannose and complex sugars) and/or high-mannose sugars.

\section{Evaluation of cell-vector binding to DC-SIGN}

Two experiments were performed to investigate whether SVGmu does in fact recognize and bind to human DCSIGN and whether binding of SVGmu-bearing LVs (FUGW/SVGmu) to 293T.DCSIGN, a stable 293T cell line expressing human DCSIGN, is enhanced by the addition of DMJ to vector-producing cells. In the first experiment, 293T.DCSIGN cells were fixed with 4\% paraformaldehyde followed by incubation with fresh supernatant containing FUGW/SVGmu. The cell-vector complexes were then stained for SVGmu and analyzed by flow cytometry. As expected, the vector produced with DMJ bound more readily to the cells, as shown by the higher percentage of SVGmu staining (Figure 5A).

Next, a radioactive-labeling assay was used to test the cell-vector binding responses for LVs produced with and without DMJ, with an additional comparison to control 293T cells, which lacked DC-SIGN expression. 293T and 293T.DCSIGN cells were seeded onto 96-well plates overnight. The cells were then washed by PBS and incubated with concentrated, ${ }^{35}$ S-labeled LVs for one hour. A second wash with PBS removed unbound vectors and the cells were lysed before they were pipetted into scintillation vials for analysis. A mannose inhibition assay was also employed to test the dependency of vector binding on the mannose- rich structures of the envelope glycoprotein. Mannose was incubated with the cells prior to the addition of LVs to block the binding sites that are mannose-linked. A much higher amount of radioactivity was detected in FUGW/ SVGmu(DMJ+) incubated with 293T.DCSIGN as compared to FUGW/SVGmu(DMJ-) (Figure 5B). In contrast, DMJ did not greatly alter the binding ability of FUGW/ SVGmu to 293T cells; vectors produced with and without DMJ resulted in a low incidence of binding. Although the addition of mannose lowered the amount of cell-vector binding measured with both types of LVs (DMJ+ and DMJ-) and both types of cells (293T.DCSIGN and 293T), it had the most significant effect on the binding of FUGW/ SVGmu(DMJ+) to the 293T.DCSIGN cells. This data suggests that the enhanced ability of the LV to bind to DCSIGN we observed was the result of its greater availability of high-mannose structures, and that this interaction can be blocked by competitive inhibition with mannose.

\section{Transduction of cells with LVs produced with DMJ}

LVs produced with DMJ were used to transduce 293T and 293T.DCSIGN cells to test the effect of the improved binding on cell transduction. Fresh vector supernatants were added to cells of each type, followed by spin-infection. The cells were then analyzed for GFP expression by flow cytometry after three days of incubation. A p24 ELISA assay was also performed to ensure that the concentrations of the different viruses were comparable. FUGW/SVGmu(DMJ+) transduced 293T. DCSIGN cells with the greatest efficiency (25.34\%) more than three-folds higher than the FUGW/SVGmu (DMJ-) vector (7.45\%) (Figure 6A). Both types of LVs had a similar amount of transduction for the control 293T cells. These results validate our expectation that more highly infective targeting vectors can be produced with the addition of DMJ in the cell culture media during vector production.

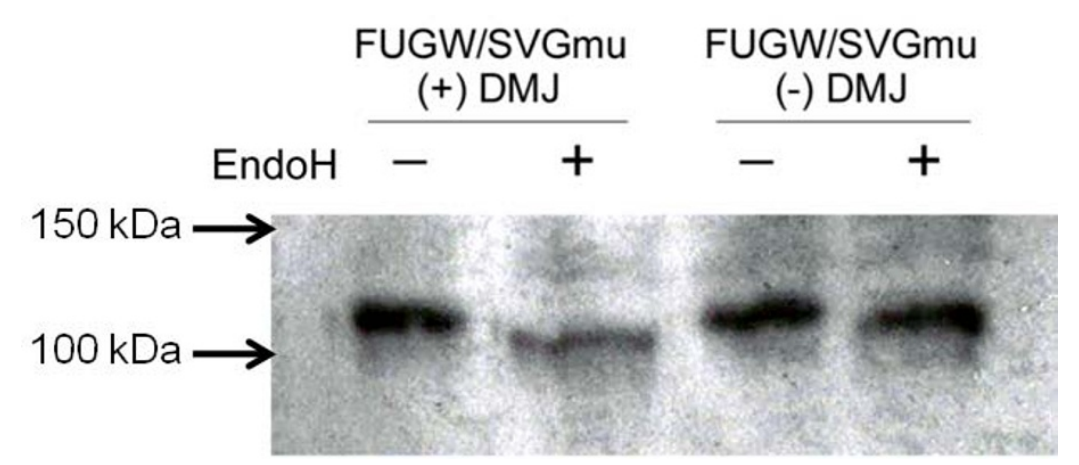

Figure 4 LVs produced in DMJ contain more high-mannose structures. Vectors were produced with or without DMJ, concentrated, and digested by EndoH, an enzyme that cleaves high-mannose structures. A western blot analysis of the undigested and digested vectors showed that the vectors produced in DMJ had a lower molecular weight after EndoH digestion compared to that of the vectors produced without DMJ. These results infer that the vector produced with DMJ had more high-mannose structures than the vector produced without DMJ. 


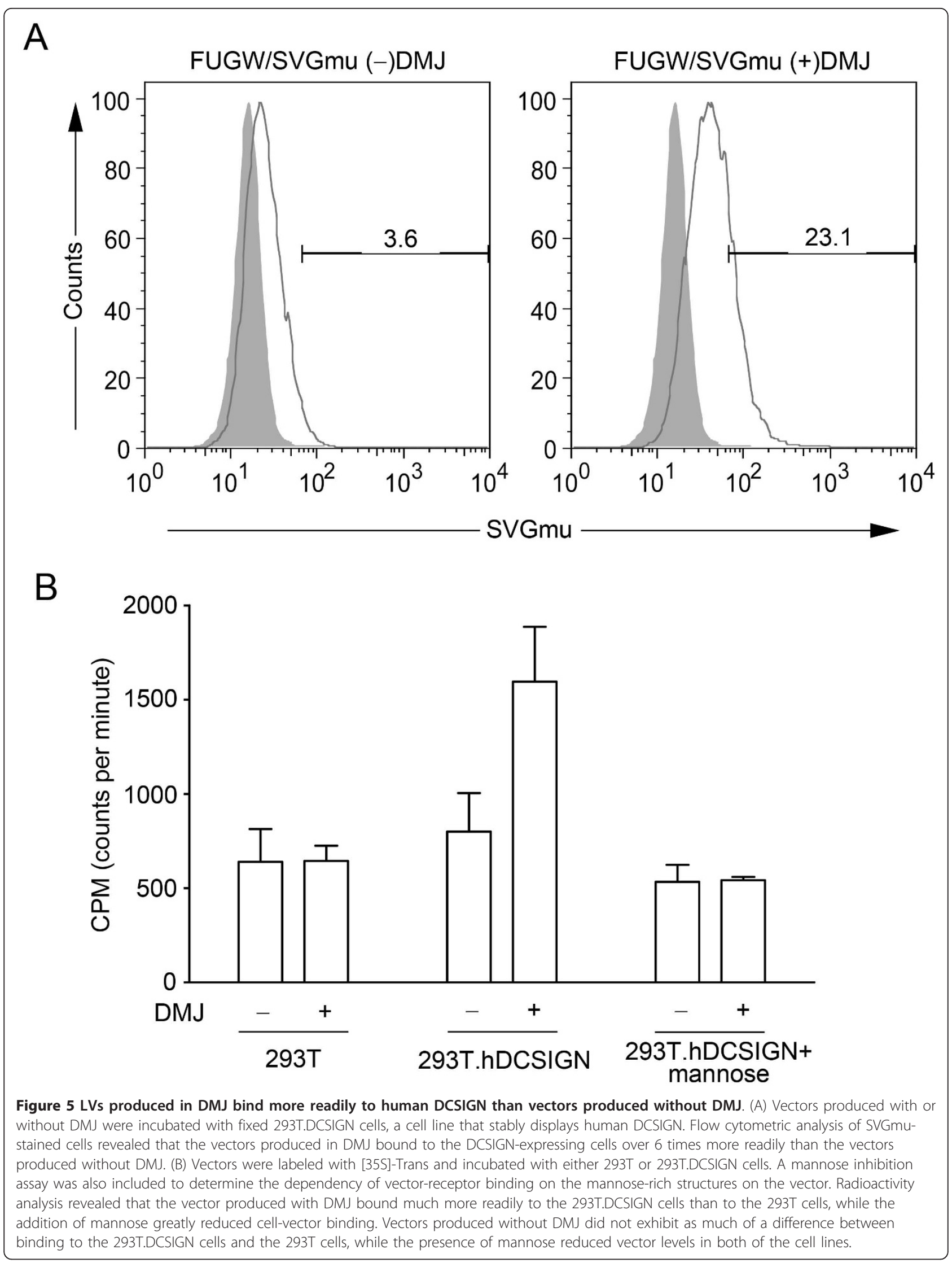




\section{A}
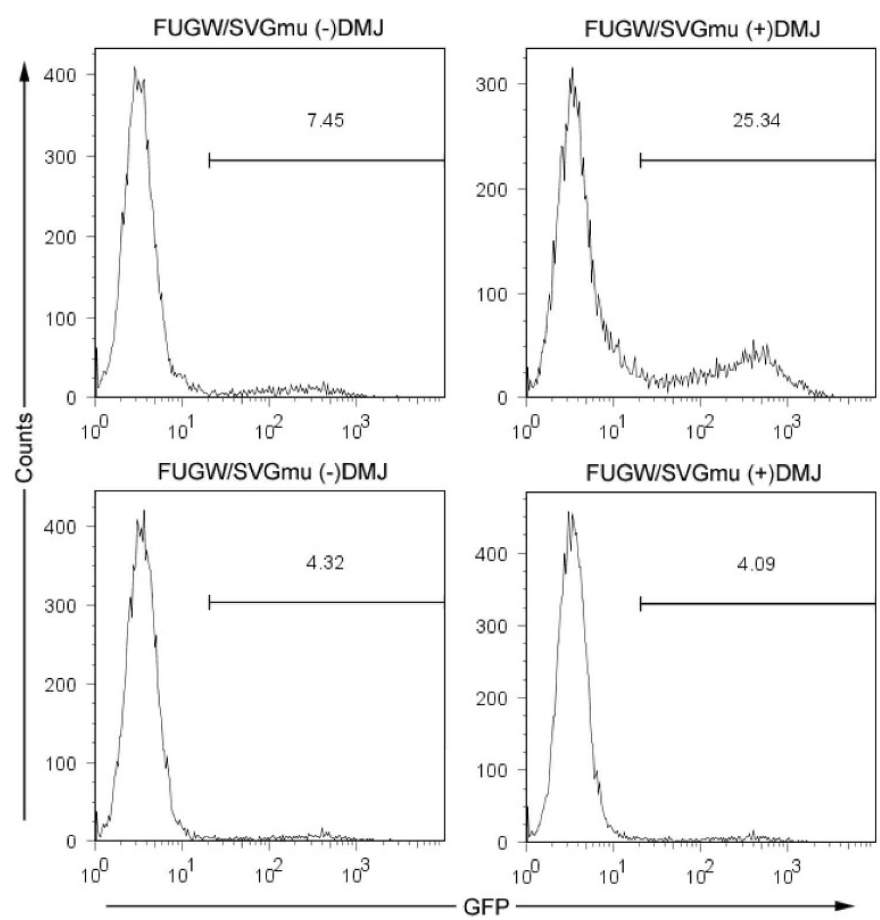

293T.DCSIGN

$293 T$
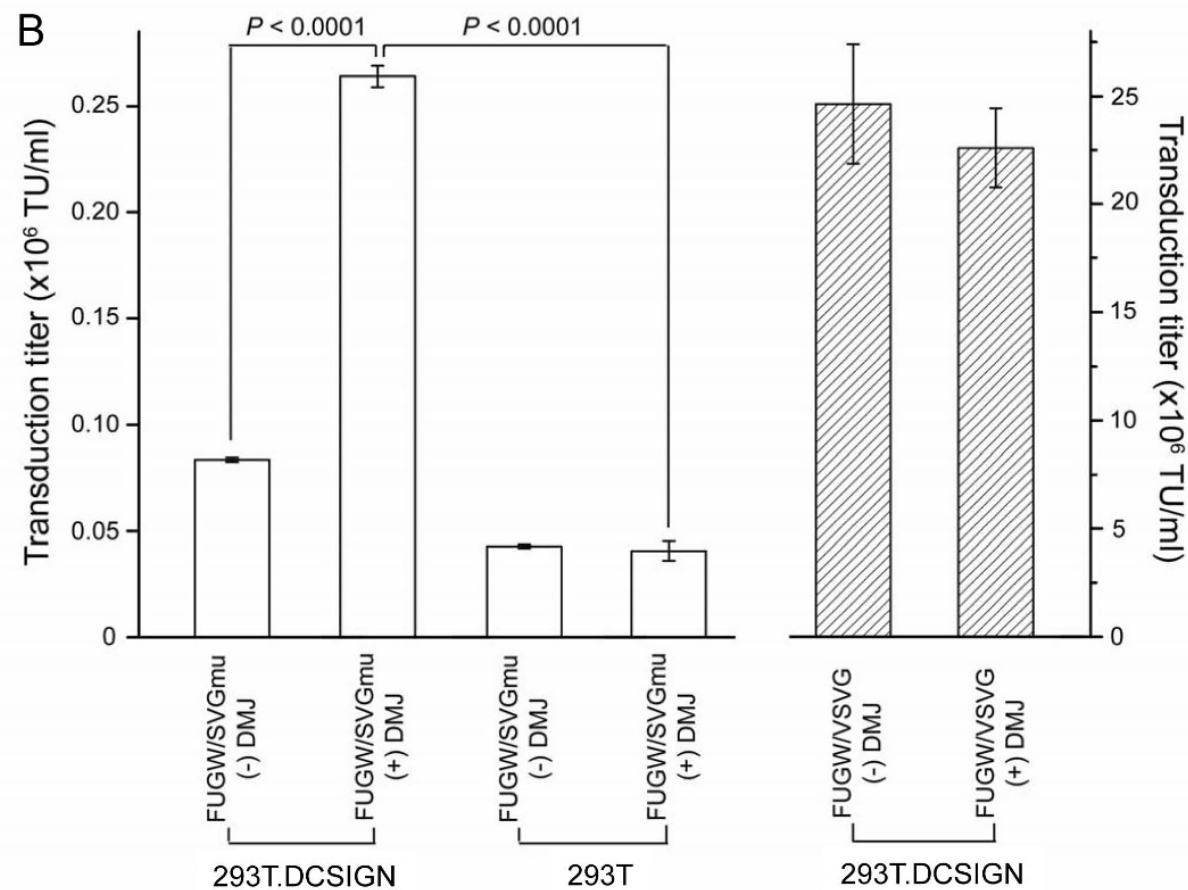

293T.DCSIGN

Figure 6 LVs produced with DMJ transduced DCSIGN-expressing cells much more efficiently than LVs produced without DMJ. (A) LVs were produced either with or without DMJ and spin-infected with 293T and 293T.DCSIGN cells. Although FUGW/SVGmu(DMJ-) preferentially transduced 293T.DCSIGN cells, FUGW/SVGmu(DMJ+) was over three-fold more efficient in transducing 293T.DCSIGN cells while maintaining similar levels of background transduction to 293T cells. (B) LVs produced with DMJ yielded higher vector titers for DCSIGN-expressing cells compared with LVs produced without DMJ. Vector titers for both FUGW/SVGmu +/- DMJ were analyzed for 293T.DCSIGN and 293T cells. On 293T.DCSIGN cells, FUGW/SVGmu(DMJ+) yielded titers over three-folds higher than those of FUGW/SVGmu(DMJ-), while titers for 293T cells for both vectors were similarly low. FUGWNSVG +/- DMJ was included as a control. 
Vector titers were then calculated for FUGW/SVGmu $+/-$ DMJ against 293T and 293T.DCSIGN cells. FUGW/ VSVG +/- DMJ were also included as controls. Triplicate experiments were performed, where cells were spin-infected with various fresh vector supernatants that had been serially diluted. Three days later, the cells were washed and analyzed for GFP expression by flow cytometry. As expected, FUGW/SVGmu(DMJ+) yielded a higher transduction titer with 293T.DCSIGN cells, with an average value of $0.26 \times 10^{6} \mathrm{TU} / \mathrm{ml}$, as compared to FUGW/SVGmu(DMJ-), which had an average transduction titer of $0.08 \times 10^{6} \mathrm{TU} / \mathrm{ml}$ (Figure 6B). FUGW/ SVGmu +/- DMJ both transduced 293T cells similarly with titers of $\sim 0.04 \times 10^{6} \mathrm{TU} / \mathrm{ml}$. As an additional control, 293T.DCSIGN cells were also tranduced by FUGW/VSVG +/- DMJ and titers of $22.60 \times 10^{6} \mathrm{TU} / \mathrm{ml}$ and $24.60 \times 10^{6} \mathrm{TU} / \mathrm{ml}$, respectively, were obtained. Thus, our titer measurement confirms that FUGW/ SVGmu(DMJ+) transduced 293T.DCSIGN cells three times more efficiently than FUGW/SVGmu(DMJ-) did, and that both FUGW/SVGmu +/- DMJ transduced $293 \mathrm{~T}$ cells at an equally low rate. The addition of DMJ to the production of the FUGW/VSVG vector did not significantly alter the vector titer against 293T.DCSIGN.

One group demonstrated that LVs pseudotyped with a similarly modified Sindbis virus envelope glycoprotein produced without DMJ treatment did not bind to DCSIGN and target DC-SIGN-positive cells [40]. The mutations used in their envelope protein were identical except for the addition of a $\mathrm{ZZ}$ domain versus the HA tag employed in our system. However, previous studies in our laboratory have shown that SVGmu binds with DC-SIGN and targets transduction to DC-SIGN-positive cells [28]. Also, compared with our data for wild-type Sindbis envelope-bearing LVs, their pseudotyped vectors are less infectious (Virus-receptor mediated transduction of dendritic cells by lentiviruses enveloped with glycoproteins derived from Semliki Forest virus and Ross River virus, submitted). Perhaps these differences in infectious vector production are indicative of an abundance of non-infectious particles that conceal interactions between the glycoprotein and DC-SIGN. Other differences, such as our use of a clonally-expanded DCSIGN-expressing cell line and other experimental settings, may further contribute to the reported inability to observe increased transduction efficiencies for DC-SIGN bearing cell lines with SVGmu envelope-bearing LVs produced without DMJ.

\section{Transduction of a DC cell line with vectors produced with} DMJ

To test the transduction efficiency of vectors produced with and without DMJ on a closer model of dendritic cells, we utilized a DC cell line (MUTZ-3 cells) that had been previously shown to closely mirror the behavior of human dendritic cells and are capable of being differentiated to express human DC-SIGN [41,42]. MUTZ-3 cells were cultured and differentiated for 7 days to express DC-SIGN (Figure 7A) before they were transduced by concentrated FUGW/SVGmu vectors produced with or without DMJ. Consistent with the results from 293T.DCSIGN transduction, the targeting vector produced with DMJ transduced the MUTZ-3 cells more efficiently $(87.4 \%)$ than the vector produced without DMJ (62.8\%) (Figure 7B). Thus, the targeting vector produced with DMJ, which contained more high-mannose structures, was able to transduce both the $293 \mathrm{~T}$. DCSIGN cell line and a human DC cell line more efficiently than the vector produced without DMJ.

\section{Conclusions}

DC-based vaccines have great potential as a new means to fight challenging diseases, such as cancers, HIV, and autoimmune diseases. Optimization of the efficiency of the viral vectors used to modify DCs could greatly strengthen this approach and make it an even more powerful tool for developing novel treatment modalities against various diseases. Our study aimed to examine the effects of DMJ, which indirectly increases the amount of high-mannose structures present on glycoproteins through the inhibition of class I $\alpha 1,2$-mannosidase activity in vector-producing cells, on the efficiency of SVGmu-bearing LV transduction of DC-SIGNexpressing cells.

We were able to conclude that the FUGW/SVGmu vector made in the presence of DMJ did in fact have increased high-mannose structures over those made without DMJ present. Our results also suggested that binding of SVGmu to DC-SIGN is directly related to amount of high-mannose structures on SVGmu, and that these interactions can be blocked by competitive inhibition. Furthermore, we found that production of the targeting vector in DMJ resulted in a three-fold increase in transduction efficiency in target cells compared to vectored produced without DMJ. Lastly, the targeting vector produced in the presence of DMJ was able to transduce MUTZ-3 cells, a cell line that has been shown to closely mimic the behavior of human peripheral blood mononuclear cells (PBMCs) and can be differentiated to cells displaying DC-SIGN, more efficiently than the vector produced without DMJ, which strengthens our belief that an increase in high-mannose structures on the targeting virus surface would result in higher transduction efficiency of human DCs.

Further investigation into the vector entry mechanism of SVGmu-pseudotyped LVs is currently being conducted using confocal microscopy with the aid of drug treatments. Greater understanding of the pathway of 


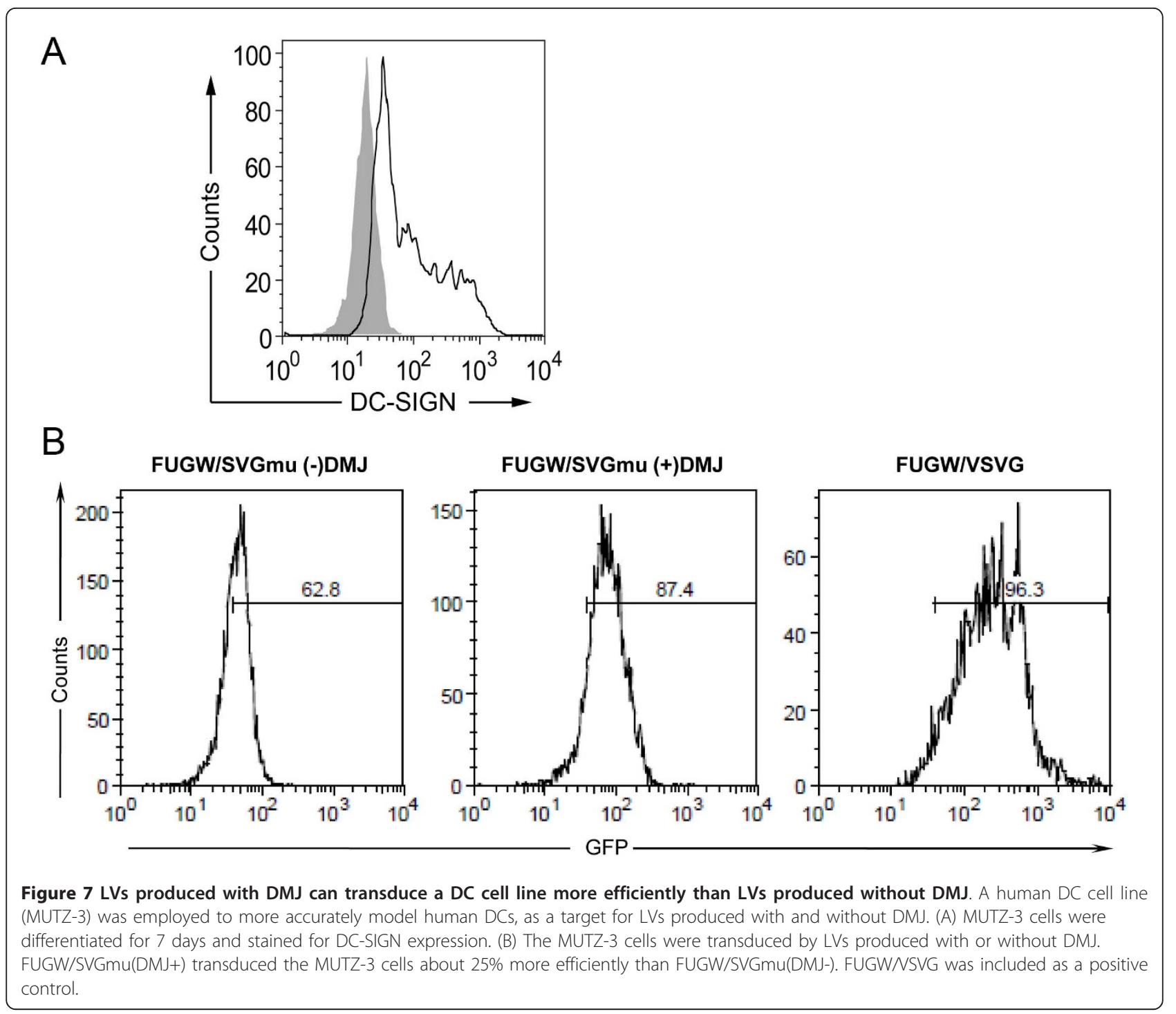

viral uptake and core release into the cytosol will facilitate the design of more effective and efficient engineered gene delivery vehicles. Additionally, siRNA can be applied to silence mannosidase in vector-producing cells to eliminate the need for DMJ in large-scale productions of optimized high-mannose-containing engineered LVs.

\section{Methods}

\section{Production of lentiviral vectors}

293T cells were transiently transfected with a standard calcium phosphate precipitation protocol. 293T cells were seeded onto $6-\mathrm{cm}$ tissue culture dishes and transfected with $5 \mu \mathrm{g}$ of the lentiviral transfer vector plasmid (FUGW or FUW), $2.5 \mu \mathrm{g}$ of the envelope plasmid (SVGmu or VSVG), and packaging plasmids (pMDLg/ pRRE and pRSV-Rev). D10 media (Dulbecco's modified Eagle's medium (Mediatech Inc., Manassas, VA) with
$10 \%$ fetal bovine serum (Sigma, St. Louis, MO), $2 \mathrm{mM}$ L-glutamine (Hyclone, Logan, UT), $100 \mathrm{U} / \mathrm{ml}$ penicillin, and $100 \mu \mathrm{g} / \mathrm{ml}$ streptomycin) with and without DMJ was replaced $4 \mathrm{~h}$ later. After $48 \mathrm{~h}$ post-transfection, the vector supernatants were harvested and filtered through a $0.45-\mu \mathrm{m}$ filter (Corning, Acton, MA). To concentrate the vector, the supernatants were ultracentrifugated (Optima L-80 K preparative ultracentrifuge, Beckman Coulter, Brea, CA) after filtration at $50,000 \times \mathrm{g}$ for 90 min. The viral pellets were resuspended in $100 \mu \mathrm{l}$ of cold PBS.

\section{Confocal imaging of lentiviral vectors}

LVs were produced with the addition of $2.5 \mu \mathrm{g}$ of a plasmid encoding GFP-Vpr. Vector supernatant was placed on polylysine-coated coverslips in 6-well culture dishes and centrifuged at $3,700 \times \mathrm{g}$ at $4^{\circ} \mathrm{C}$ for $2 \mathrm{~h}$ with a Sorvall 
Legend RT centrifuge (DJB Labcare, Buckinghamshire, England). The coverslips were then washed twice with cold PBS and immunostained with anti-HA-biotin antibody (Miltenyi Biotec, Bergisch Gladbach, Germany) and Cy5-streptavidin (Invitrogen, Carlsbad, CA). The samples were fluorescently imaged by a Zeiss LSM 510 laser scanning confocal microscope with filter sets for fluorescein and $\mathrm{Cy} 5$ and a plan-apochromat oil immersion objective $(63 \times / 1.4)$.

\section{Vector digestion by EndoH and western blot}

$7 \mu \mathrm{l}$ of concentrated virus was combined with $2 \mu \mathrm{l}$ of lysis buffer and incubated at $37^{\circ} \mathrm{C}$ for $30 \mathrm{~min}$. Next, the glycoprotein was denatured by adding $1 \mu \mathrm{l}$ of $10 \times$ Glycoprotein Denaturing Buffer to the mixture and heating the reaction to $100^{\circ} \mathrm{C}$ for $10 \mathrm{~min}$. The protein was then digested by EndoH by adding $2 \mu \mathrm{l}$ of $10 \times$ G5 Reaction Buffer, $5 \mu \mathrm{l}$ of EndoH (NEB, Ipswich, MA), and $3 \mu \mathrm{l}$ of $\mathrm{H}_{2} \mathrm{O}$. This reaction was incubated at $37^{\circ} \mathrm{C}$ for $1 \mathrm{~h}$. Protein gels were run and then western blots were performed to transfer the proteins onto membranes. The membranes were then blocked by $5 \%$ milk with TBST at $4^{\circ} \mathrm{C}$ for $1 \mathrm{~h}$. The membranes were washed with TBST and then stained for anti-HA-biotin antibody for $1 \mathrm{~h}$. They were then washed again, stained for streptavidinHRP (R\&D Systems, Minneapolis, MN) for $1 \mathrm{hr}$, and washed. To develop the western blot, TMB solution was spread onto the surface of the membrane and left for $10 \mathrm{~min}$ at room temperature. The membrane was then imaged.

\section{Radioactive labeling and mannose inhibition of lentiviral vectors}

293T cells were transfected by vector plasmids as described earlier, without the addition of DMJ. $20 \mathrm{~h}$ post-transfection, the media was replaced with methionine-free D10 with or without DMJ. After $4 \mathrm{~h}, 50 \mu \mathrm{l}$ of [35S]-Trans (MP Biochemicals, Solon, OH) was added to $15-\mathrm{cm}$ tissue culture dishes and incubated at $37^{\circ} \mathrm{C}$. $48 \mathrm{~h}$ post-tranfection, viral supernatants were harvested, filtered, and concentrated. For the mannose inhibition assay, $10 \mathrm{mM}$ of D-mannose was added to the $293 \mathrm{~T}$. hDCSIGN cells prior to the cell-virus binding tests.

\section{Vector transduction of cells}

293T or 293T.DCSIGN cells were seeded into a 24-well culture dish at $0.2 \times 10^{6}$ cells per well and spin-infected with $1 \mathrm{ml}$ of viral supernatant per well at 2,500 rpm and $25^{\circ} \mathrm{C}$ for 90 min using a Sorvell Legend centrifuge. The cell supernatants were then replaced with fresh D10 media and incubated at $37^{\circ} \mathrm{C}$ for 3 days with $5 \% \mathrm{CO}_{2}$. FACS analysis was used to determine the percentage of GFP+ cells present. To determine transduction titers, the dilution ranges that showed a linear response were used.

\section{Transduction of a human DC cell line}

MUTZ-3 cells (Deutsche Sammlung von Mikroorganismen und Zellkulturen, Braunschweig, Germany) were cultured in 24-well tissue culture plates in $\alpha$ MEM media (BioWhittaker, Walkersville, MD) with 20\% FBS (Sigma-Aldrich, St. Louis, MO) and $40 \mathrm{ng} / \mathrm{mL}$ GM-CSF (PeproTech, Rocky Hill, NJ). To differentiate the cells to express human DC-SIGN, MUTZ-3 cells were cultured in the presence of IL- 4 and GM-CSF $(100 \mathrm{ng} / \mathrm{mL}$ of each; PeproTech) for 7 days. FACS analysis of cells stained with anti-human DCSIGN-PE (BioLegend, San Diego, CA) confirmed the presence of the human DCSIGN marker. The cells $\left(1 \times 10^{5}\right)$ were spin-infected with concentrated virus and the medium was replaced with fresh medium containing IL-4 and GM-CSF. The cells were analyzed by flow cytometry for GFP expression 3 days post-tranduction.

\section{Abbreviations}

DC): dendritic cell; (DMJ): 1-deoxymannojirimycin; (FUGW): lentiviral vector carrying a GFP reporter gene under the control of the human ubiquitin $\mathrm{C}$ promoter; (GFP): green fluorescent protein; (GFPVpr): GFP fused to the Nterminus of Vpr; (HS): heparin sulfate; (LV): lentiviral vector; (MHC): major histocompatibility complex; (SVG): Sindbis virus glycoprotein; (SVGmu): engineered SVG; (Vpr): the HIV accessory protein viral protein R; (VSVG): vesicular stomatitis viral glycoprotein.

\section{Acknowledgements}

We thank Chi-Lin Lee for the preparation of figures. This work was supported by a National Institute of Health grant R01-Al068978.

\section{Authors' contributions}

AT and PW designed experiments. AT, SF, and KJ carried out all the experiments. AT and PW wrote the manuscript. All authors read and approved the final manuscript.

\section{Competing interests}

The authors declare that they have no competing interests.

Received: 5 October 2010 Accepted: 28 January 2011

Published: 28 January 2011

\section{References}

1. Mellman I, Steinman RM: Dendritic cells: specialized and regulated antigen processing machines. Cell 2001, 106:255-258.

2. Steinman RM, Banchereau J: Taking dendritic cells into medicine. Nature 2007, 449:419-426.

3. Banchereau J, Steinman RM: Dendritic cells and the control of immunity. Nature 1998, 392:245-252

4. Banchereau J, Briere F, Caux C, Davoust J, Lebecque S, Liu YJ, Pulendran B, Palucka K: Immunobiology of dendritic cells. Annual review of immunology 2000, 18:767-811.

5. Figdor CG, de Vries IJ, Lesterhuis WJ, Melief CJ: Dendritic cell immunotherapy: mapping the way. Nat Med 2004, 10:475-480.

6. Breckpot K, Heirman C, Neyns B, Thielemans K: Exploiting dendritic cells for cancer immunotherapy: genetic modification of dendritic cells. J Gene Med 2004, 6:1175-1188.

7. Ribas A, Butterfield LH, Glaspy JA, Economou JS: Cancer immunotherapy using gene-modified dendritic cells. Current gene therapy 2002, 2:57-78. 
8. Draper SJ, Heeney JL: Viruses as vaccine vectors for infectious diseases and cancer. Nat Rev Microbiol 2010, 8:62-73.

9. Gardlik R, Palffy R, Hodosy J, Lukacs J, Turna J, Celec P: Vectors and delivery systems in gene therapy. Med Sci Monit 2005, 11:RA110-121.

10. Schaffer DV, Koerber JT, Lim KI: Molecular engineering of viral gene delivery vehicles. Annu Rev Biomed Eng 2008, 10:169-194.

11. Song W, Kong HL, Carpenter H, Torii H, Granstein R, Rafii S, Moore MA, Crystal RG: Dendritic cells genetically modified with an adenovirus vector encoding the CDNA for a model antigen induce protective and therapeutic antitumor immunity. J Exp Med 1997, 186:1247-1256.

12. Esslinger C, Chapatte L, Finke D, Miconnet I, Guillaume P, Levy F, MacDonald HR: In vivo administration of a lentiviral vaccine targets DCs and induces efficient CD8(+) T cell responses. J Clin Invest 2003, 111:1673-1681.

13. Palmowski MJ, Lopes L, Ikeda Y, Salio M, Cerundolo V, Collins MK: Intravenous injection of a lentiviral vector encoding NY-ESO-1 induces an effective CTL response. J Immunol 2004, 172:1582-1587.

14. Pincha M, Sundarasetty BS, Stripecke R: Lentiviral vectors for immunization: an inflammatory field. Expert Rev Vaccines 2010, 9:309-321.

15. He Y, Munn D, Falo LD Jr: Recombinant lentivector as a genetic immunization vehicle for antitumor immunity. Expert Rev Vaccines 2007, 6:913-924.

16. Lopes L, Dewannieux M, Gileadi U, Bailey R, Ikeda Y, Whittaker C, Collin MP, Cerundolo V, Tomihari M, Ariizumi K, Collins MK: Immunization with a lentivector that targets tumor antigen expression to dendritic cells induces potent CD8+ and CD4+ T-cell responses. J Virol 2008, 82:86-95.

17. Follenzi A, Battaglia M, Lombardo A, Annoni A, Roncarolo MG, Naldini L. Targeting lentiviral vector expression to hepatocytes limits transgenespecific immune response and establishes long-term expression of human antihemophilic factor IX in mice. Blood 2004, 103:3700-3709.

18. Tacken PJ, de Vries IJ, Torensma R, Figdor CG: Dendritic-cell immunotherapy: from ex vivo loading to in vivo targeting. Nat Rev Immunol 2007, 7:790-802

19. Soilleux EJ, Morris LS, Leslie G, Chehimi J, Luo Q, Levroney E, Trowsdale J, Montaner LJ, Doms RW, Weissman D, et al: Constitutive and induced expression of DC-SIGN on dendritic cell and macrophage subpopulations in situ and in vitro. J Leukoc Biol 2002, 71:445-457.

20. Lozach PY, Amara A, Bartosch B, Virelizier JL, Arenzana-Seisdedos F, Cosset FL, Altmeyer R: C-type lectins L-SIGN and DC-SIGN capture and transmit infectious hepatitis C virus pseudotype particles. J Biol Chem 2004, 279:32035-32045

21. Figdor CG, van Kooyk Y, Adema GJ: C-type lectin receptors on dendritic cells and Langerhans cells. Nat Rev Immunol 2002, 2:77-84

22. Soilleux EJ: DC-SIGN (dendritic cell-specific ICAM-grabbing non-integrin) and DC-SIGN-related (DC-SIGNR): friend or foe? Clin Sci (Lond) 2003, 104:437-446

23. Lozach PY, Burleigh L, Staropoli I, Amara A: The C type lectins DC-SIGN and L-SIGN: receptors for viral glycoproteins. Methods Mol Biol 2007 379:51-68.

24. Klimstra WB, Nangle EM, Smith MS, Yurochko AD, Ryman KD: DC-SIGN and L-SIGN can act as attachment receptors for alphaviruses and distinguish between mosquito cell- and mammalian cell-derived viruses. J Virol 2003, 77:12022-12032.

25. Morizono K, Bristol G, Xie YM, Kung SK, Chen IS: Antibody-directed targeting of retroviral vectors via cell surface antigens. J Virol 2001, 75:8016-8020.

26. Cronin J, Zhang $X Y$, Reiser J: Altering the tropism of lentiviral vectors through pseudotyping. Current gene therapy 2005, 5:387-398

27. Morizono K, Xie Y, Ringpis GE, Johnson M, Nassanian H, Lee B, Wu L, Chen IS: Lentiviral vector retargeting to P-glycoprotein on metastatic melanoma through intravenous injection. Nat Med 2005, 11:346-352

28. Yang L, Yang H, Rideout K, Cho T, Joo Kl, Ziegler L, Elliot A, Walls A, Yu D, Baltimore D, Wang P: Engineered lentivector targeting of dendritic cells for in vivo immunization. Nat Biotechnol 2008, 26:326-334.

29. Dai B, Yang L, Yang H, Hu B, Baltimore D, Wang P: HIV-1 Gag-specific immunity induced by a lentivector-based vaccine directed to dendritic cells. Proc Natl Acad Sci USA 2009, 106:20382-20387.

30. Hu B, Dai B, Wang P: Vaccines delivered by integration-deficient lentiviral vectors targeting dendritic cells induces strong antigen-specific immunity. Vaccine 2010, 28:6675-6683.
31. Mitchell DA, Fadden AJ, Drickamer K: A novel mechanism of carbohydrate recognition by the C-type lectins DC-SIGN and DC-SIGNR. Subunit organization and binding to multivalent ligands. J Biol Chem 2001, 276:28939-28945.

32. Geiitenbeek TB, Kwon DS, Torensma R, van Vliet SJ, van Duijnhoven GC, Middel J, Cornelissen IL, Nottet HS, KewalRamani VN, Littman DR, et al: DCSIGN, a dendritic cell-specific HIV-1-binding protein that enhances transinfection of T cells. Cell 2000, 100:587-597.

33. Feinberg $H$, Mitchell DA, Drickamer $K$, Weis WI: Structural basis for selective recognition of oligosaccharides by DC-SIGN and DC-SIGNR. Science 2001, 294:2163-2166.

34. Elbein AD: Glycosidase inhibitors: inhibitors of $\mathrm{N}$-linked oligosaccharide processing. FASEB J 1991, 5:3055-3063.

35. Elbein AD, Legler G, Tlusty A, McDowell W, Schwarz R: The effect of deoxymannojirimycin on the processing of the influenza viral glycoproteins. Arch Biochem Biophys 1984, 235:579-588.

36. Vallee F, Karaveg K, Herscovics A, Moremen KW, Howell PL: Structural basis for catalysis and inhibition of $\mathrm{N}$-glycan processing class I alpha 1,2mannosidases. J Biol Chem 2000, 275:41287-41298.

37. Balzarini J: The alpha(1,2)-mannosidase I inhibitor 1-deoxymannojirimycin potentiates the antiviral activity of carbohydrate-binding agents against wild-type and mutant HIV-1 strains containing glycan deletions in gp120. FEBS Lett 2007, 581:2060-2064.

38. Lois C, Hong EJ, Pease S, Brown EJ, Baltimore D: Germline transmission and tissue-specific expression of transgenes delivered by lentiviral vectors. Science 2002, 295:868-872.

39. Joo Kl, Wang P: Visualization of targeted transduction by engineered lentiviral vectors. Gene Ther 2008, 15:1384-1396.

40. Morizono K, Ku A, Xie Y, Harui A, Kung SK, Roth MD, Lee B, Chen IS: Redirecting lentiviral vectors pseudotyped with Sindbis virus-derived envelope proteins to DC-SIGN by modification of $\mathrm{N}$-linked glycans of envelope proteins. J Virol 2010, 84:6923-6934.

41. Larsson $\mathrm{K}$, Lindstedt $\mathrm{M}$, Borrebaeck CA: Functional and transcriptional profiling of MUTZ-3, a myeloid cell line acting as a model for dendritic cells. Immunology 2006, 117:156-166.

42. Santegoets SJ, van den Eertwegh AJ, van de Loosdrecht AA, Scheper RJ, de Gruijl TD: Human dendritic cell line models for DC differentiation and clinical DC vaccination studies. J Leukoc Biol 2008, 84:1364-1373.

doi:10.1186/1754-1611-5-1

Cite this article as: Tai et al:: Production of lentiviral vectors with enhanced efficiency to target dendritic cells by attenuating mannosidase activity of mammalian cells. Journal of Biological Engineering 2011 5:1.

\section{Submit your next manuscript to BioMed Central and take full advantage of:}

- Convenient online submission

- Thorough peer review

- No space constraints or color figure charges

- Immediate publication on acceptance

- Inclusion in PubMed, CAS, Scopus and Google Scholar

- Research which is freely available for redistribution

Submit your manuscript at www.biomedcentral.com/submit
C Biomed Central 